\title{
Rapid thermal co-annihilation through bound states in QCD
}

\author{
Seyong $\operatorname{Kim}^{a}$ and M. Laine ${ }^{b}$ \\ ${ }^{a}$ Department of Physics, Sejong University, \\ Seoul 143-747, South Korea \\ ${ }^{b}$ AEC, Institute for Theoretical Physics, University of Bern, \\ Sidlerstrasse 5, CH-3012 Bern, Switzerland \\ E-mail: skim@sejong.ac.kr, laine@itp.unibe.ch
}

ABSTRACT: The co-annihilation rate of heavy particles close to thermal equilibrium, which plays a role in many classic dark matter scenarios, can be "simulated" in QCD by considering the pair annihilation rate of a heavy quark and antiquark at a temperature of a few hundred $\mathrm{MeV}$. We show that the so-called Sommerfeld factors, parameterizing the rate, can be defined and measured non-perturbatively within the NRQCD framework. Lattice measurements indicate a modest suppression in the octet channel, in reasonable agreement with perturbation theory, and a large enhancement in the singlet channel, much above the perturbative prediction. The additional enhancement is suggested to originate from bound state formation and subsequent decay. Making use of a Green's function based method to incorporate thermal corrections in perturbative co-annihilation rate computations, we show that qualitative agreement with lattice data can be found once thermally broadened bound states are accounted for. We suggest that our formalism may also be applicable to specific dark matter models which have complicated bound state structures.

KeYwords: Thermal Field Theory, Cosmology of Theories beyond the SM, Heavy Quark Physics, Lattice QCD

ARXIV EPRINT: 1602.08105 


\section{Contents}

1 Introduction 1

2 Setup 2

2.1 Physics background 2

2.2 Basic definitions 4

3 Reduction to a static 2-point correlator $\quad 7$

3.1 Absorptive parts of 4-quark operators in imaginary time 7

$\begin{array}{lll}3.2 & \text { Canonical analysis and analytic continuation } & 8\end{array}$

$\begin{array}{lll}3.3 & \text { Wick contractions } & 11\end{array}$

4 Perturbative estimates 12

$\begin{array}{llr}5 & \text { Lattice analysis } & 15\end{array}$

$\begin{array}{llr}6 & \text { Discussion and outlook } & 18\end{array}$

$\begin{array}{lr}\text { A Heavy quark propagators } & \mathbf{2 0}\end{array}$

\section{Introduction}

Heavy particle co-annihilation represents a subtle problem, because the slowly moving particles experience strong initial state effects before the final inelastic process. Reversing the time direction, the same can be said about pair creation. Nevertheless, within two-body quantum mechanics in a system with a Coulomb potential, this physics was understood long ago $[1,2]$. It also plays an essential role for heavy particle pair creation in QCD [3]. The enhancement or suppression of the annihilation rate, depending on whether the Coulomb interaction is attractive or repulsive, is generically referred to as the Sommerfeld effect.

If the co-annihilating particles are part of a thermal medium, the Sommerfeld effect plays a role in a modified form. In this case the velocities of the annihilating particles are not fixed, but come from a statistical distribution. In particular, if we assume the heavy particles to be in kinetic equilibrium, the velocities are distributed according to the Boltzmann weight. Then we speak of thermally averaged Sommerfeld factors. The thermally averaged Sommerfeld factors are relevant for cosmology, particularly for determining the abundance of heavy weakly interacting dark matter particle species [4-7], which decouple deep in the non-relativistic regime. A recent example of an embedding of the corresponding Sommerfeld factors in a realistic setting can be found in ref. [8].

The starting point of the present paper is the observation that physics formally similar to dark matter co-annihilation takes place with heavy quarks and antiquarks in hot QCD 
at a temperature of a few hundred $\mathrm{MeV}$. Going beyond a leading-order computation based on Boltzmann equations [9-11], the rate of their chemical equilibration can be defined on a general level [12], and can be shown to be sensitive to the physics of the thermal Sommerfeld effect [13]. Even though the associated time scale appears to be too long to play a practical role within the life-time $\sim 10 \mathrm{fm} / \mathrm{c}$ of a fireball generated in a heavy-ion collision experiment, this analogy nevertheless means that established methods of QCD, including those of lattice QCD, can be used to investigate the problem. In particular, in ref. [13] a strategy was outlined for implementing in Euclidean spacetime the absorptive parts of 4-quark operators, which can be used for describing the decays of quark-antiquark states within the NRQCD effective theory framework [14, 15].

The purpose of the present paper is to realize this proposal. A main part is to develop further the theoretical formulation, showing that the measurement can be reduced to 2point functions and that analytic continuation back to Minkowskian spacetime poses no problem. We also carry out an exploratory lattice study, finding an intriguing pattern with an enhancement in the singlet channel much larger than predicted by the standard formulae used in the literature for incorporating thermal Sommerfeld enhancement. An explanation for this observation in terms of bound-state physics is put forward.

The plan of this paper is the following. After defining the basic observables in section 2, we show in section 3, through a spectral representation and a canonical analysis, how their measurement can be related to purely static 2-point correlation function ratios. The perturbative evaluation of these correlators is discussed in section 4, and corresponding lattice measurements are reported in section 5. A concluding discussion and an outlook are offered in section 6 . Readers not interested in the details of the theoretical and lattice analyses are suggested to consult section 2.1 and subsequently proceed to sections 4 and 6 .

\section{Setup}

\subsection{Physics background}

In order to outline the physics problem in a simple setting, we consider heavy quarks and antiquarks, with a pole mass $M$, placed in a heat bath at a temperature $T \ll M$. We stress that even though we make use of QCD terminology, the discussion until eq. (2.4) applies rather generally, with the gauge group replaced as appropriate. In the QCD context the only specific assumption made is that we consider time scales up to some thousands of fm/c, so that the weak decays of the heavy quarks can be omitted; this can be viewed as the analogue of "R-parity conservation" in supersymmetric theories. Thereby the only number-changing reactions are pair annihilations and creations.

In the heavy-quark limit, QCD has an "emergent" symmetry, in that the quark and antiquark numbers are conserved separately. This conservation is only violated by higherdimensional operators suppressed by $1 / M$. Omitting such operators it is possible to define separate distribution functions for heavy quarks and antiquarks. We assume that both are close to kinetic and chemical equilibrium, whereby the distribution functions have the

forms $f_{p}=\bar{f}_{p} \approx 2 N_{\mathrm{c}} \exp \left(-E_{p} / T\right)$, where $E_{p} \equiv \sqrt{p^{2}+M^{2}}$ and $2 N_{\mathrm{c}}$ counts the spin and 
colour degrees of freedom. Here $p \equiv|\mathbf{p}|$ is the spatial momentum with respect to the heat bath.

Consider now the total number density, $n=\int_{\mathbf{p}}\left(f_{p}+\bar{f}_{p}\right)+n_{\text {bound }}$, where $n_{\text {bound }}$ denotes the density of quark-antiquark bound states. The equilibrium value of the total density reads $n_{\text {eq }} \approx 4 N_{\mathrm{c}} \int_{\mathbf{p}} \exp \left(-E_{p} / T\right)+\mathcal{O}\left(e^{-2 M / T}\right)$, where the last term indicates the bound state contribution. If the equilibrium conditions are evolving, for instance through a Hubble expansion characterized by a rate $H$, then the system attempts to adjust its number density to this change. Within a Boltzmann equation approach the evolution equation has the form $[16]$

$$
\left(\partial_{t}+3 H\right) n \approx-c\left(n^{2}-n_{\mathrm{eq}}^{2}\right) .
$$

If we linearize the right-hand side around equilibrium, this can be rewritten as

$$
\left(\partial_{t}+3 H\right) n=-\Gamma_{\text {chem }}\left(n-n_{\text {eq }}\right)+\mathcal{O}\left(n-n_{\text {eq }}\right)^{2} .
$$

The coefficient $\Gamma_{\text {chem }}=2 c n_{\mathrm{eq}}$ is called the chemical equilibration rate. It tells how efficiently the system is able to re-adjust its density towards the evolving $n_{\text {eq }}$, and encodes the effects of the microscopic processes which can change the quark and antiquark number densities, notably pair creations and annihilations. Note that, unlike eq. (2.1), the form of eq. (2.2) represents a general linearization and is thus valid beyond the Boltzmann approach.

Let us stress that $\Gamma_{\text {chem }}$ describes the slow evolution of a number density, averaged over a large volume. The associated frequency scale is $\omega \sim \Gamma_{\text {chem }} \sim T \exp (-M / T) \ll T$. This slow evolution is caused by infinitely many rare individual processes. The individual processes are associated with pair creations and annihilations and carry a large energy, $E \sim 2 M \gg T$. We are not studying these individual reactions separately; in fact the energy scale $2 M$ can be integrated out whereby the system can be described by an effective theory known as the NRQCD $[14,15]$, capturing physics at scales $|E-2 M| \ll M$.

Now, it is important to realize that even though bound states give a strongly suppressed contribution to $n_{\text {eq }}$, they give an enhanced contribution to $\Gamma_{\text {chem }}$. Indeed, as already mentioned, in $n_{\mathrm{eq}} \sim e^{-M / T}$ the bound state contribution is $n_{\text {bound }} \sim e^{-2 M / T}$. But $\Gamma_{\text {chem }}$ originates from reactions where a quark and antiquark come together, i.e. $\left|\partial_{t} n\right| \sim e^{-2 M / T}$. In a bound state the quark and antiquark are "already" together, and with a less suppressed Boltzmann weight, because of a binding energy $\Delta E>0$. Therefore the decay rate from bound states is

$$
\left|\partial_{t} n_{\text {bound }}\right| \sim e^{-(2 M-\Delta E) / T} .
$$

If $T \lesssim \Delta E \sim M \alpha_{\mathrm{s}}^{2}$, this contribution dominates the annihilation rate. This leads to a physical picture in which chemical equilibration proceeds via a "two-stage" process: in thermal equilibrium, only a small fraction of quarks and antiquarks form bound states. But it is those bound states which are most efficiently depleted as the temperature decreases. Bound state formation itself is a fast process, without any exponential suppression factors.

Even though the potential significance of bound states has been mentioned in many cosmological studies, such as refs. [17, 18], they have not established themselves as a standard ingredient in quantitative estimates of the thermal freeze-out process. One good 
reason is certainly that bound states only exist in very specific weakly interacting models. But there is also another issue, namely that the computations are usually based on the Boltzmann equation, eq. (2.1), where $c=\langle\sigma v\rangle$ is a thermally averaged annihilation cross section. Thereby, if not sufficient care is taken, the basic assumption that the system can be described by dilute single-particle on-shell states may be inadvertently built into the formalism from the beginning. Recently the importance of bound-state effects has been stressed in, e.g., refs. [19-23], which also suggested various ways to include them. The simplest possibility is to add a bound state phase space distribution as an additional variable in a set of Boltzmann equations (even though it is not clear whether a thermally broadened resonance can be accurately treated as an on-shell degree of freedom). Here we follow a different avenue, aiming eventually to offer for a framework permitting to scrutinize the accuracy of the Boltzmann approach. For this purpose, we take the general linearization of eq. (2.2) as a starting point.

\subsection{Basic definitions}

We next recall how $\Gamma_{\text {chem }}$ can be defined, through a linear response type analysis, as a transport coefficient [12], and how its subsequent evaluation reveals the presence of a Sommerfeld effect [13]. We only give the main steps, referring to refs. [12, 13] for details.

Let $\psi$ denote the Dirac spinor of a heavy quark. Making use of a representation in which $\gamma^{0}=\operatorname{diag}\left(\mathbb{1}_{2 \times 2},-\mathbb{1}_{2 \times 2}\right), \psi$ can be expressed in terms of spinors which have two non-zero components:

$$
\theta \equiv \frac{1}{2}\left(\mathbb{1}+\gamma^{0}\right) \psi, \quad \chi \equiv \frac{1}{2}\left(\mathbb{1}-\gamma^{0}\right) \psi .
$$

In the following the vanishing components are omitted. The spinor $\theta$ can be associated with a quark state, and the conjugate of the spinor $\chi$ with an antiquark state. In particular the energy density carried by heavy quarks and antiquarks, which for large $M$ is equal to their rest mass times their number density, can be described through

$$
H \equiv M\left(\theta^{\dagger} \theta-\chi^{\dagger} \chi\right)=M\left(\theta_{\alpha i}^{*} \theta_{\alpha i}+\chi_{\alpha i} \chi_{\alpha i}^{*}\right)
$$

where $\theta_{\alpha i}$ annihilates a quark of colour $\alpha$ and spin $i, \chi_{\alpha i}^{*}$ does the same for an antiquark, and $M \equiv M_{\text {rest }}$ is the heavy quark rest mass. ${ }^{1}$ Note that we treat eq. (2.5), without any terms suppressed by $1 / M$, as a definition; up to a normalization, it represents a Noether charge density related to the NRQCD Lagrangian.

We are concerned with the imaginary-time 2-point correlator of the operator $H$,

$$
\Delta(\tau) \equiv \int_{\mathbf{x}}\langle H(\tau, \mathbf{x}) H(0, \mathbf{0})\rangle, \quad 0<\tau<\frac{1}{T} .
$$

\footnotetext{
${ }^{1}$ In perturbative considerations, particularly in section 4, we make no difference between rest and kinetic masses, assuming that both correspond to a pole mass. It is worth noting that the precise definition in eq. (2.5) is irrelevant since the mass cancels in eq. (2.12); only the kinetic mass is relevant for the Sommerfeld factors to be defined presently. However the rest mass strongly affects the quark number susceptibility $\chi_{f}$, appearing e.g. in eqs. (2.17) and (2.20), and it also appears trivially in eqs. (2.7) and (2.9).
} 
Given that $H$ is a conserved charge density, $\Delta(\tau)$ is constant within the standard NRQCD theory [14]. In full QCD, however, the number of heavy quarks is not conserved, because heavy quarks and antiquarks can pair annihilate into gluons and light quarks. These processes can be described by adding 4-quark operators to the NRQCD Lagrangian [15].

The 4-quark operators can be classified according to the gauge and spin quantum numbers of the 2-quark "constituent operators" of which they are composed (the 4-quark operators themselves are gauge singlets and Lorentz scalars). We carry out the main discussion in terms of the "singlet" operator,

$$
\mathcal{O}_{1}\left({ }^{1} S_{0}\right) \equiv \theta^{\dagger} \chi \chi^{\dagger} \theta, \quad \delta S_{\mathrm{M}}=\frac{f_{1}\left({ }^{1} S_{0}\right) \mathcal{O}_{1}\left({ }^{1} S_{0}\right)}{M^{2}}
$$

whose coefficient has an "absorptive" imaginary part at $\mathcal{O}\left(\alpha_{\mathrm{s}}^{2}\right)$ :

$$
\operatorname{Im} f_{1}\left({ }^{1} S_{0}\right)=\frac{C_{\mathrm{F}}}{2 N_{\mathrm{c}}} \pi \alpha_{\mathrm{s}}^{2}, \quad C_{\mathrm{F}} \equiv \frac{N_{\mathrm{c}}^{2}-1}{2 N_{\mathrm{c}}} .
$$

For future reference let us also write down one of the octet operators,

$$
\mathcal{O}_{8}\left({ }^{1} S_{0}\right) \equiv \theta^{\dagger} T^{a} \chi \chi^{\dagger} T^{a} \theta, \quad \delta S_{\mathrm{M}}=\frac{f_{8}\left({ }^{1} S_{0}\right) \mathcal{O}_{8}\left({ }^{1} S_{0}\right)}{M^{2}},
$$

where $T^{a}$ are generators of $\mathrm{SU}\left(N_{\mathrm{c}}\right)$, normalized as $\operatorname{Tr}\left(T^{a} T^{b}\right)=\delta^{a b} / 2$. We work to leading non-trivial order in $\alpha_{\mathrm{s}}(2 M)$ whereby $\mathcal{O}\left(\alpha_{\mathrm{s}}^{3}\right)$ corrections to the coefficients (cf. e.g. ref. [24]) as well as operators suppressed by the relative velocity $v^{2} \sim \alpha_{\mathrm{s}}^{2}$ (cf. e.g. ref. [25]) are omitted.

If the singlet operator is added to the NRQCD Lagrangian, and the correlator of eq. (2.6) is computed within this theory, then $\Delta(\tau)$ is no longer a constant. We assume that the correlator is computed to first order as an expansion in $\operatorname{Im} f_{1}\left({ }^{1} S_{0}\right) / M^{2}$. If the result is Fourier decomposed,

$$
\tilde{\Delta}\left(\omega_{n}\right)=\int_{0}^{\beta} \mathrm{d} \tau e^{i \omega_{n} \tau} \Delta(\tau), \quad \beta \equiv \frac{1}{T},
$$

and a corresponding spectral function is determined, $\rho_{\Delta}(\omega)=\operatorname{Im} \tilde{\Delta}\left(\omega_{n} \rightarrow-i\left[\omega+i 0^{+}\right]\right)$, then a "transport coefficient" can be defined as

$$
\Omega_{\mathrm{chem}} \equiv \lim _{\omega \ll T} 2 T \omega \rho_{\Delta}(\omega) .
$$

Note that the transport coefficient is extracted from a $1 / \omega$ tail of the spectral function, rather than from a linear slope as is sometimes the case; the reason is that the interaction responsible for the process considered has been treated as an insertion. The heavy quark chemical equilibration rate is subsequently obtained from [12]

$$
\Gamma_{\text {chem }}=\frac{\Omega_{\text {chem }}}{2 \chi_{f} M^{2}}
$$

where $\chi_{f}$ is the quark-number susceptibility related to the heavy flavour. Note that $M$ appears linearly in $H$ (cf. eq. (2.5)) and quadratically in $\Omega_{\text {chem }}$ (cf. eq. (2.6)) and therefore drops out in this ratio. 
The singlet operator in eq. (2.7) mediates decays which experience the so-called Sommerfeld enhancement [1-3]. Within perturbation theory, omitting bound state effects, the quark-number susceptibility $\chi_{f}$ and the singlet contribution to $\Omega_{\text {chem }}$, denoted by $\delta_{1} \Omega_{\text {chem }}$, read [13]

$$
\begin{array}{r}
\chi_{f} \approx 4 N_{\mathrm{c}} \int_{\mathbf{p}} e^{-\beta E_{p}} \approx 4 N_{\mathrm{c}}\left(\frac{M T}{2 \pi}\right)^{\frac{3}{2}} e^{-M / T}, \\
\delta_{1} \Omega_{\mathrm{chem}} \approx 32 N_{\mathrm{c}} \operatorname{Im} f_{1}\left({ }^{1} S_{0}\right) \int_{\mathbf{p}, \mathbf{q}} e^{-\beta\left(E_{p}+E_{q}\right)} \bar{S}_{1},
\end{array}
$$

where $\bar{S}_{1}$ is a thermal Sommerfeld factor, related to a vacuum Sommerfeld factor $S_{1}$ by

$$
\bar{S}_{1} \simeq \frac{\int_{\mathbf{v}} e^{-\beta E_{\mathrm{rel}}} S_{1}(v)}{\int_{\mathbf{v}} e^{-\beta E_{\mathrm{rel}}}} .
$$

Here $E_{\text {rel }} \equiv M v^{2}$, with $v=|\mathbf{v}|$, is the energy of the relative motion of the annihilating particles. The normalization is such that without any resummation, $\bar{S}_{1}=S_{1}=1$.

When higher-order perturbative corrections to $S_{1}$ are considered, there is particular subseries of them which proceeds in powers of $\alpha_{\mathrm{s}} / v$. These are summed to all orders into the Sommerfeld factor $S_{1}$. After the thermal average in eq. (2.15), $v$ is parametrically of order $\sqrt{T / M}$. Therefore the thermal Sommerfeld effect is important for $T \lesssim \alpha_{\mathrm{s}}^{2} M$, whereas it evaluates to unity for $T \gg \alpha_{\mathrm{s}}^{2} M$.

Inspired by eqs. (2.13) and (2.14), we may define a non-perturbative Sommerfeld factor as

$$
\bar{S}_{1} \equiv \frac{N_{\mathrm{c}} \delta_{1} \Omega_{\mathrm{chem}}}{2 \operatorname{Im} f_{1}\left({ }^{1} S_{0}\right) \chi_{f}^{2}},
$$

where $\delta_{1} \Omega_{\text {chem }}$ is to be evaluated with the operator in eq. (2.7) inserted to first order. Note that the coefficient $\operatorname{Im} f_{1}\left({ }^{1} S_{0}\right)$ drops out in this ratio because $\delta_{1} \Omega_{\text {chem }}$ is linear in it. With this definition the chemical equilibration rate from eq. (2.12) can be expressed as

$$
\delta_{1} \Gamma_{\text {chem }}=\frac{\operatorname{Im} f_{1}\left({ }^{1} S_{0}\right)}{M^{2}} \times \chi_{f} \times \frac{\bar{S}_{1}}{N_{\mathrm{c}}} .
$$

Thereby the physical result has factorized into a high-energy part $\operatorname{Im} f_{1}\left({ }^{1} S_{0}\right)$ as well as ingredients which can be addressed non-perturbatively. These are $\bar{S}_{1}$, which can be extracted from a dimensionless ratio of correlation functions (cf. eq. (3.17)), and the susceptibility $\chi_{f}$, which is a standard observable measuring essentially the equilibrium density $n_{\text {eq }}$. The appearance of $1 / N_{\mathrm{c}}$ in eq. (2.17) is a consequence of including $N_{\mathrm{c}}$ in $\chi_{f}$.

For the octet channel, the analogue of eq. (2.14) reads [13]

$$
\delta_{8} \Omega_{\mathrm{chem}} \approx 16\left(N_{\mathrm{c}}^{2}-1\right) \operatorname{Im} f_{8}\left({ }^{1} S_{0}\right) \int_{\mathbf{p}, \mathbf{q}} e^{-\beta\left(E_{p}+E_{q}\right)} \bar{S}_{8}
$$

where the perturbative value of $\bar{S}_{8}$ is given by an average like in eq. (2.15). We now define the non-perturbative value through

$$
\bar{S}_{8} \equiv \frac{N_{\mathrm{c}}^{2} \delta_{8} \Omega_{\mathrm{chem}}}{\left(N_{\mathrm{c}}^{2}-1\right) \operatorname{Im} f_{8}\left({ }^{1} S_{0}\right) \chi_{f}^{2}} .
$$


For the chemical equilibration rate this leads to

$$
\delta_{8} \Gamma_{\text {chem }}=\frac{\operatorname{Im} f_{8}\left({ }^{1} S_{0}\right)}{M^{2}} \times \chi_{f} \times \frac{\left(N_{\mathrm{c}}^{2}-1\right) \bar{S}_{8}}{2 N_{\mathrm{c}}^{2}} .
$$

The colour factors follow from the definition of the octet operator in eq. (2.9) and from the inclusion of one $N_{\mathrm{c}}$ in $\chi_{f}$. In QCD there is also another octet operator at $\mathcal{O}\left(\alpha_{\mathrm{s}}^{2}, v^{0}\right)$, whose coefficient $\operatorname{Im} f_{8}\left({ }^{3} S_{1}\right)$ is proportional to the number of light flavours $N_{\mathrm{f}}$ [15]; for brevity we concentrate on spin-independent operators here but do not expect any qualitative changes from spin-dependent terms.

\section{Reduction to a static 2-point correlator}

When the correlator in eq. (2.6) is evaluated by inserting the operator of eq. (2.7) to first order, we are faced with a 3 -point function. We start by recalling that in the imaginary-time formalism the 3 -point function splits up into a 4-point function. However, we subsequently show that the 4-point function can be reduced to a simple 2-point function, if we are only interested in extracting the transport coefficient $\Omega_{\text {chem }}$ and not the full spectral shape.

\subsection{Absorptive parts of 4-quark operators in imaginary time}

The imaginary parts of the 4-fermion vertices in NRQCD represent pair annihilations of heavy quarks and antiquarks. Going to the center-of-mass frame, the vertex is a function of the total energy $E$ of the annihilating pair. Expanding the energy dependence in powers of $E-2 M \approx M v^{2}$, where $v$ denotes the velocity of $q$ in the $q \bar{q}$ rest frame, the leading term is a constant, i.e. of $\mathcal{O}\left(v^{0}\right)$. We restrict to the leading order in the non-relativistic expansion and thereby only consider the constant term.

Even though the term is constant, the corresponding vertex is non-local in imaginary time [12]. In general, the imaginary part corresponds to a spectral function, or cut, of a certain Green's function. More specifically, the 4 -fermion vertex can be viewed as a limit of a correlator of two quark-antiquark operators. Given a spectral function for the latter, $\rho(E)$, the corresponding imaginary-time correlator is given by

$$
G(\tau)=\int_{0}^{\infty} \frac{\mathrm{d} E}{\pi} \rho(E) f_{\mathrm{B}}(E)\left[e^{(\beta-|\tau|) E}+e^{|\tau| E}\right], \quad-\beta<\tau<\beta,
$$

where $f_{\mathrm{B}}$ is the Bose distribution. Given that we know $\rho$ only for $E \approx 2 M$, we cut off the low- $E$ contribution by a mass scale $\Lambda$, lying parametrically in the range $T \ll \Lambda \ll 2 M$. Denoting the constant value by $\rho_{0}$, we may then estimate

$$
G(\tau) \approx \int_{\Lambda}^{\infty} \frac{\mathrm{d} E}{\pi} \rho_{0} f_{\mathrm{B}}(E)\left[e^{(\beta-|\tau|) E}+e^{|\tau| E}\right] \approx \frac{\rho_{0}}{\pi} \frac{e^{-|\tau| \Lambda}}{|\tau|} \approx \frac{\rho_{0}}{\pi|\tau|},
$$

where we approximated $|\tau| \sim 1 /(2 M) \ll \beta$, $\exp (-\Lambda / T) \ll 1$, and $|\tau| \Lambda \sim \Lambda /(2 M) \ll 1$. Therefore, rather than obtaining $\delta(t)$ as would be the case in real time, we now get a 
non-locality $1 /|\tau|$. Specifically, the imaginary part of the singlet operator of eq. (2.7) can be expressed as a real effective operator in a Euclidean action,

$$
S_{\mathrm{E}} \approx-\frac{\operatorname{Im} f_{1}\left({ }^{1} S_{0}\right)}{\pi M^{2}} \int_{0}^{\beta} \mathrm{d} \tau_{1} \int_{0}^{\beta} \mathrm{d} \tau_{2} \int_{\mathbf{x}} \frac{\left(\theta^{\dagger} \chi\right)\left(\tau_{1}, \mathbf{x}\right)\left(\chi^{\dagger} \theta\right)\left(\tau_{2}, \mathbf{x}\right)}{\left|\tau_{1}-\tau_{2}\right|}
$$

We remark that for the argumentation in eq. (3.2) it was essential that $1 /|\tau|$ originated from the contribution of large energies and corresponds therefore to a short separation. In eq. (3.3) this restriction has been lifted; this is not a concern because, as our subsequent analysis shows, the contribution still effectively emerges from short separations (cf. eq. (3.14)). A related point is that it is not clear how eq. (3.3) should be defined at the contact point $\tau_{1}=\tau_{2}$. Fortunately, the contact point is naturally regularized by the considerations to follow, so for the moment we simply treat eq. (3.3) as a formal construction.

\subsection{Canonical analysis and analytic continuation}

Expanding now $\exp \left(-S_{\mathrm{E}}\right) \rightarrow 1-S_{\mathrm{E}}$, defining

$$
\Delta(\tau) \equiv \frac{\operatorname{Im} f_{1}\left({ }^{1} S_{0}\right)}{\pi M^{2}} \mathcal{E}(\tau),
$$

and making use of translational invariance in order to adjust the spatial coordinates, eqs. (2.6) and (3.3) imply that we are faced with the 4-point correlator

$$
\mathcal{E}(\tau) \equiv \int_{\mathbf{x}, \mathbf{y}} \int_{0}^{\beta} \mathrm{d} \tau_{2} \int_{0}^{\beta} \mathrm{d} \tau_{1} \frac{\left\langle H(\tau, \mathbf{x}) H(0, \mathbf{y})\left(\theta^{\dagger} \chi\right)\left(\tau_{1}, \mathbf{0}\right)\left(\chi^{\dagger} \theta\right)\left(\tau_{2}, \mathbf{0}\right)\right\rangle}{\left|\tau_{1}-\tau_{2}\right|}
$$

We proceed to giving a canonical interpretation to eq. (3.5), showing that the 4-point correlator reduces to a 2-point correlator once we extract the corresponding transport coefficient.

We reiterate, first of all, that within the NRQCD Lagrangian $\int_{\mathbf{x}} H$ corresponds to a conserved charge (particle plus antiparticle number times the rest mass). In other words, the corresponding operator commutes with the Hamiltonian $\hat{\mathcal{H}},\left[\hat{\mathcal{H}}, \int_{\mathbf{x}} \hat{H}\right]=0$. Therefore the Heisenberg operator is time independent,

$$
\int_{\mathbf{x}} \hat{H}(\tau, \mathbf{x})=\int_{\mathbf{x}} \hat{H}\left(\tau^{\prime}, \mathbf{x}\right)
$$

It turns out, however, that there are contact terms at the positions $\tau_{1}$ and $\tau_{2}$ when considering the correlator in eq. (3.5). For $\tau_{1}>\tau_{2}$, which will turn out to be the case relevant for us (see below), we need to have $\tau>\tau_{1}$ or $\tau<\tau_{2}$, otherwise the correlator vanishes up to exponentially small corrections. Therefore $\tau$ can only be chosen from the range $\tau \in\left[0, \tau_{2}\right) \cup\left(\tau_{1}, \beta\right]$

In order to re-express eq. (3.5) in the canonical formalism in this case, we recall that the Euclidean path integral corresponds to a time-ordered expectation value. Now, within the NRQCD theory, the operators $\hat{\theta}^{\dagger}$ and $\hat{\theta}$ are the creation and annihilation operators for the quark states, respectively. For the antiquark states, $\hat{\chi}$ corresponds to a creation operator and $\hat{\chi}^{\dagger}$ to an annihilation operator. Therefore $\hat{\theta}^{\dagger} \hat{\chi}$ creates a quark-antiquark state, 
and $\hat{\chi}^{\dagger} \hat{\theta}$ annihilates it. According to eq. (2.5), $\int_{\mathbf{x}} \hat{H}$ measures the rest mass of the quarks and antiquarks present.

We denote energy eigenstates involving a heavy quark and a heavy antiquark by $|q \bar{q}, m\rangle$, whereas states involving neither are denoted by $|n\rangle$. The corresponding energies are denoted by $E_{m}$ and $\epsilon_{n}$, respectively. Then $\int_{\mathbf{x}} \hat{H}|q \bar{q}, m\rangle=2 M|q \bar{q}, m\rangle$ whereas $\int_{\mathbf{x}} \hat{H}|n\rangle=0$. Note that the quark-antiquark states can be either bound states or scattering states.

Inserting now completeness relations ${ }^{2}$ and making use of eq. (3.6), the numerator of eq. (3.5) can be expressed as

$$
\begin{aligned}
\int_{\mathbf{x}, \mathbf{y}}\langle H & \left.(\tau, \mathbf{x}) H(0, \mathbf{y})\left(\theta^{\dagger} \chi\right)\left(\tau_{1}, \mathbf{0}\right)\left(\chi^{\dagger} \theta\right)\left(\tau_{2}, \mathbf{0}\right)\right\rangle\left.\right|_{\tau_{1}>\tau_{2}}\left[\theta\left(\tau-\tau_{1}\right)+\theta\left(\tau_{2}-\tau\right)\right] \\
& =\frac{1}{\mathcal{Z}} \operatorname{Tr}\left[e^{-\beta \hat{\mathcal{H}}}\left(\hat{\theta}^{\dagger} \hat{\chi}\right)\left(\tau_{1}, \mathbf{0}\right)\left(\hat{\chi}^{\dagger} \hat{\theta}\right)\left(\tau_{2}, \mathbf{0}\right) \int_{\mathbf{x}, \mathbf{y}} \hat{H}(0, \mathbf{x}) \hat{H}(0, \mathbf{y})\right] \\
& =\frac{1}{\mathcal{Z}} \sum_{m, n}\left\langle q \bar{q}, m\left|e^{-\beta \hat{\mathcal{H}}}\left(\hat{\theta}^{\dagger} \hat{\chi}\right)\left(\tau_{1}, \mathbf{0}\right)\right| n\right\rangle\left\langle n\left|\left(\hat{\chi}^{\dagger} \hat{\theta}\right)\left(\tau_{2}, \mathbf{0}\right) \int_{\mathbf{x}, \mathbf{y}} \hat{H}(0, \mathbf{x}) \hat{H}(0, \mathbf{y})\right| q \bar{q}, m\right\rangle \\
& =\frac{4 M^{2}}{\mathcal{Z}} \sum_{m, n} e^{-\beta E_{m}} e^{\left(\tau_{1}-\tau_{2}\right)\left(E_{m}-\epsilon_{n}\right)}\left\langle q \bar{q}, m\left|\hat{\theta}^{\dagger} \hat{\chi}\right| n\right\rangle\left\langle n\left|\hat{\chi}^{\dagger} \hat{\theta}\right| q \bar{q}, m\right\rangle .
\end{aligned}
$$

We have restricted to the contribution from sectors of the Hilbert space with at most one quark-antiquark state present. The quark-antiquark states had to be placed at the outer boundaries in order for $\int_{\mathbf{x}} \hat{H}$ to give a non-zero contribution.

We note that had we chosen $\tau_{2}>\tau_{1}$ instead, then the creation operator $\left(\hat{\theta}^{\dagger} \hat{\chi}\right)\left(\tau_{1}, \mathbf{0}\right)$ would have appeared to the right of the annihilation operator $\left(\hat{\chi}^{\dagger} \hat{\theta}\right)\left(\tau_{2}, \mathbf{0}\right)$. Then the states $|n\rangle$ would have to be replaced by states containing two heavy quarks and two antiquarks. This is the case irrespective of the range chosen for $\tau$, because one of the $\hat{H}$-operators is always at $\tau=0$. The corresponding contribution to $\Gamma_{\text {chem }}$ would be exponentially suppressed by $\exp (-2 M / T)$ and will be omitted.

Eq. (3.7) should clarify the physical meaning of eq. (3.5). As indicated by the matrix elements squared, we are considering the decays of quark-antiquark states into light degrees of freedom. Physically, the quark-antiquark states are assumed to be initially close to thermal equilibrium; our general framework corresponds to a linear response analysis, so that in eq. (3.7) the quark-antiquark states are in thermal equilibrium.

Understanding the time dependence of eq. (3.7) requires care. Let us denote

$$
\mathcal{C}_{m n} \equiv \frac{4 M^{2}}{\mathcal{Z}} e^{-\beta E_{m}}\left\langle q \bar{q}, m\left|\hat{\theta}^{\dagger} \hat{\chi}\right| n\right\rangle\left\langle n\left|\hat{\chi}^{\dagger} \hat{\theta}\right| q \bar{q}, m\right\rangle
$$

Recalling the restrictions on $\tau$ the function in eq. (3.5) can then be written as

$$
\mathcal{E}(\tau)=\sum_{m, n} \mathcal{C}_{m n} \int_{0}^{\beta} \mathrm{d} \tau_{2} \int_{\tau_{2}}^{\beta} \mathrm{d} \tau_{1} \frac{e^{\left(\tau_{1}-\tau_{2}\right)\left(E_{m}-\epsilon_{n}\right)}}{\tau_{1}-\tau_{2}}\left[\theta\left(\tau-\tau_{1}\right)+\theta\left(\tau_{2}-\tau\right)\right] .
$$

\footnotetext{
${ }^{2}$ For notational simplicity we assume that the system is placed for a moment in a finite volume so that the energy spectrum is discrete.
} 
Taking a time derivative; carrying out one integration with the help of the resulting Dirac $\delta$-function; and representing the denominator as $1 /\left(\tau_{1}-\tau_{2}\right)=\int_{0}^{\infty} \mathrm{d} s e^{-s\left(\tau_{1}-\tau_{2}\right)}$, we get

$$
\begin{aligned}
\mathcal{E}^{\prime}(\tau) & =-\sum_{m, n} \mathcal{C}_{m n} \int_{\tau}^{\beta-\tau} \frac{\mathrm{d} x}{x} e^{x\left(E_{m}-\epsilon_{n}\right)} \\
& =-\sum_{m, n} \mathcal{C}_{m n} \int_{\tau}^{\beta-\tau} \mathrm{d} x \int_{0}^{\infty} \mathrm{d} s e^{x\left(E_{m}-\epsilon_{n}-s\right)} \\
& =\sum_{m, n} \mathcal{C}_{m n} \int_{0}^{\infty} \mathrm{d} s \frac{e^{\tau\left(E_{m}-\epsilon_{n}-s\right)}-e^{(\beta-\tau)\left(E_{m}-\epsilon_{n}-s\right)}}{E_{m}-\epsilon_{n}-s} .
\end{aligned}
$$

This can be integrated into

$$
\mathcal{E}(\tau)=\sum_{m, n} \mathcal{C}_{m n} \int_{0}^{\infty} \mathrm{d} s \frac{e^{\tau\left(E_{m}-\epsilon_{n}-s\right)}+e^{(\beta-\tau)\left(E_{m}-\epsilon_{n}-s\right)}}{\left(E_{m}-\epsilon_{n}-s\right)^{2}}-\text { const. },
$$

where the (infinite) integration constant can be omitted, because its Fourier transform has no non-trivial cut. Going over to the normalization of eq. (3.4), a Fourier transform according to eq. (2.10) yields

$$
\tilde{\Delta}\left(\omega_{n}\right)=\frac{\operatorname{Im} f_{1}\left({ }^{1} S_{0}\right)}{\pi M^{2}} \sum_{m, n} \mathcal{C}_{m n} \int_{0}^{\infty} \mathrm{d} s \frac{e^{\beta\left(E_{m}-\epsilon_{n}-s\right)}-1}{\left(E_{m}-\epsilon_{n}-s\right)^{2}}\left[\frac{1}{i \omega_{n}+E_{m}-\epsilon_{n}-s}+\left(\omega_{n} \rightarrow-\omega_{n}\right)\right] .
$$

The corresponding spectral function reads

$$
\begin{aligned}
\rho_{\Delta}(\omega) & =\frac{\operatorname{Im} f_{1}\left({ }^{1} S_{0}\right)}{M^{2}} \sum_{m, n} \mathcal{C}_{m n} \int_{0}^{\infty} \mathrm{d} s \frac{e^{\beta\left(E_{m}-\epsilon_{n}-s\right)}-1}{\left(E_{m}-\epsilon_{n}-s\right)^{2}}\left[\delta\left(-\omega+E_{m}-\epsilon_{n}-s\right)-(\omega \rightarrow-\omega)\right] \\
\stackrel{\omega, \epsilon_{n}}{ } & \ll E_{m} \frac{\operatorname{Im} f_{1}\left({ }^{1} S_{0}\right)}{M^{2}} \sum_{m, n} \mathcal{C}_{m n} \frac{e^{\beta \omega}-e^{-\beta \omega}}{\omega^{2}},
\end{aligned}
$$

where we noted that the dynamical energy scales contained in the effective description, reflected by the energy eigenvalues $\epsilon_{n}$, are by assumption much smaller than the heavy quark-antiquark energies $E_{m} \sim 2 M$, so that the contribution emerges from $s \sim 2 M{ }^{3}$ Taking finally the limit in eq. (2.11) and inserting subsequently the value of $\mathcal{C}_{m n}$ from eq. (3.8) yields

$$
\begin{aligned}
\Omega_{\text {chem }} & =\frac{4 \operatorname{Im} f_{1}\left({ }^{1} S_{0}\right)}{M^{2}} \sum_{m, n} \mathcal{C}_{m n} \\
& =16 \operatorname{Im} f_{1}\left({ }^{1} S_{0}\right) \frac{1}{\mathcal{Z}} \sum_{m, n} e^{-\beta E_{m}}\left\langle q \bar{q}, m\left|\hat{\theta}^{\dagger} \hat{\chi}\right| n\right\rangle\left\langle n\left|\hat{\chi}^{\dagger} \hat{\theta}\right| q \bar{q}, m\right\rangle \\
& =16 \operatorname{Im} f_{1}\left({ }^{1} S_{0}\right) \frac{1}{\mathcal{Z}} \operatorname{Tr}\left[e^{-\beta \hat{\mathcal{H}}}\left(\hat{\theta}^{\dagger} \hat{\chi}\right)\left(0^{+}, \mathbf{0}\right)\left(\hat{\chi}^{\dagger} \hat{\theta}\right)(0, \mathbf{0})\right] \\
& =16 \operatorname{Im} f_{1}\left({ }^{1} S_{0}\right)\left\langle\left(\theta^{\dagger} \chi\right)\left(0^{+}, \mathbf{0}\right)\left(\chi^{\dagger} \theta\right)(0, \mathbf{0})\right\rangle .
\end{aligned}
$$

\footnotetext{
${ }^{3}$ This is a subtle point. It could be said that the energies $E_{m} \sim 2 M$ had already been integrated out in order to arrive at the NRQCD description. However, in order to get the correct limit, we need to keep the rest mass in the heavy quark Lagrangian here (cf. eq. (A.3)); it can only be shown a posteriori (see below) that the rest mass can be shifted away also in our finite-temperature observables.
} 


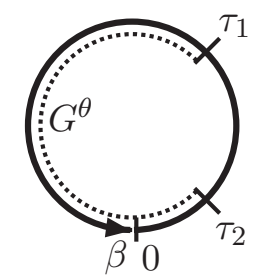

Figure 1. The propagator $G^{\theta}\left(\tau_{2}, \mathbf{0} ; \tau_{1}, \mathbf{0}\right)$ for the "unnatural" time ordering $\tau_{1}>\tau_{2}$. In this case the propagator represents a movement across the point $\tau=\beta$. The circle stands for the imaginary time direction. At zero temperature $(\beta \rightarrow \infty)$ the propagator would be exponentially suppressed. This structure corresponds to eq. (3.7), where only light parton states appear between $\tau_{2}$ and $\tau_{1}$.

Here an essential point was that there was no functional dependence on the energy eigenvalues $\epsilon_{n}$, so that we could re-identify the sum $\sum_{n}|n\rangle\langle n|$ as a unit operator. Subsequently we returned to a path integral representation. We also indicated one of the time arguments by $0^{+}$in order to maintain the correct ordering.

Eq. (3.14) is one of our main results. Effectively, it represents the expectation value of the imaginary part of the singlet operator in eq. (2.7), albeit in a spacetime with a Euclidean signature, with a periodic time direction, and with a specific time ordering. Eq. (3.14) shows that even though the general representation of the absorptive parts of 4-quark operators is cumbersome (cf. section 3.1), the final transport coefficient can be extracted from an "almost" local 4-fermion operator. The time ordering in eq. (3.14), represented by the time argument $0^{+}$, has however a specific consequence, to which we now turn.

\subsection{Wick contractions}

In order to express eq. (3.14) in terms of propagators, we need to recall some of their basic properties. The heavy quark propagator is defined in appendix A. An important consequence of the fact that non-relativistic propagators are defined by equations which are of first order in time is that $G^{\theta}\left(\tau_{2}, \mathbf{x} ; \tau_{1}, \mathbf{y}\right)=\left\langle\theta\left(\tau_{2}, \mathbf{x}\right) \theta^{\dagger}\left(\tau_{1}, \mathbf{y}\right)\right\rangle$ is obtained by integrating into the region $\tau_{2}>\tau_{1}$ (cf. eq. (A.3)), and $G^{\chi}\left(\tau_{2}, \mathbf{x} ; \tau_{1}, \mathbf{y}\right)=\left\langle\chi\left(\tau_{2}, \mathbf{x}\right) \chi^{\dagger}\left(\tau_{1}, \mathbf{y}\right)\right\rangle$ is obtained by integrating into the region $\tau_{1}>\tau_{2}$. However in eq. (3.14) the time argument of $\theta^{\dagger}$ is larger than that of $\theta$, and the time argument of $\chi$ is larger than that of $\chi^{\dagger}$. Therefore a nonzero contraction requires integrating around the imaginary-time direction. The situation is illustrated in figure 1 .

With these observations and the relations in eqs. (A.5) and (A.6) in mind, the correlator in eq. (3.14) can be expressed as

$$
\begin{aligned}
\left\langle\left(\theta^{\dagger} \chi\right)\left(0^{+}, \mathbf{0}\right)\left(\chi^{\dagger} \theta\right)(0, \mathbf{0})\right\rangle & =-\left\langle G_{\alpha \gamma ; i j}^{\theta}\left(0, \mathbf{0} ; 0^{+}, \mathbf{0}\right) G_{\gamma \alpha ; j i}^{\chi}\left(0^{+}, \mathbf{0} ; 0, \mathbf{0}\right)\right\rangle \\
& =-\left\langle G_{\alpha \gamma ; i j}^{\theta}\left(\beta, \mathbf{0} ; 0^{+}, \mathbf{0}\right) G_{\gamma \alpha ; j i}^{\chi}\left(0^{+}, \mathbf{0} ; \beta, \mathbf{0}\right)\right\rangle \\
& =\operatorname{Tr}\left\langle G^{\theta}(\beta, \mathbf{0} ; 0, \mathbf{0}) G^{\theta \dagger}(\beta, \mathbf{0} ; 0, \mathbf{0})\right\rangle,
\end{aligned}
$$

where $\alpha, \gamma \in\left\{1, \ldots, N_{\mathrm{c}}\right\}$ are colour indices, and $i, j \in\{1,2\}$ are spin indices. We may also express $\chi_{f}$, entering eq. (2.16), in the same notation. A few manipulations, including the 
use of eq. (A.6), lead to

$$
\begin{aligned}
\chi_{f} & =\int_{\mathbf{x}}\left\langle\left(\theta^{\dagger} \theta+\chi^{\dagger} \chi\right)(\tau, \mathbf{x})\left(\theta^{\dagger} \theta+\chi^{\dagger} \chi\right)(0, \mathbf{0})\right\rangle \\
& =-\int_{\mathbf{x}} \operatorname{Tr}\left\langle G^{\theta}(0, \mathbf{0} ; \tau, \mathbf{x}) G^{\theta}(\tau, \mathbf{x} ; 0, \mathbf{0})+G^{\chi}(0, \mathbf{0} ; \tau, \mathbf{x}) G^{\chi}(\tau, \mathbf{x} ; 0, \mathbf{0})\right\rangle \\
& =\int_{\mathbf{x}} \operatorname{Tr}\left\langle G^{\theta}(\beta, \mathbf{0} ; \tau, \mathbf{x}) G^{\theta}(\tau, \mathbf{x} ; 0, \mathbf{0})+G^{\chi}(0, \mathbf{0} ; \tau, \mathbf{x}) G^{\chi}(\tau, \mathbf{x} ; \beta, \mathbf{0})\right\rangle \\
& =\operatorname{Tr}\left\langle G^{\theta}(\beta, \mathbf{0} ; 0, \mathbf{0})+G^{\theta \dagger}(\beta, \mathbf{0} ; 0, \mathbf{0})\right\rangle=2 \operatorname{Re} \operatorname{Tr}\left\langle G^{\theta}(\beta, \mathbf{0} ; 0, \mathbf{0})\right\rangle .
\end{aligned}
$$

Inserting eq. (3.15) into eq. (3.14) and normalizing the result according to eq. (2.16), with $\chi_{f}$ inserted from (3.16), we get our final result for the non-perturbative Sommerfeld factor:

$$
\bar{S}_{1}=\frac{\frac{1}{2 N_{\mathrm{c}}} \operatorname{Tr}\left\langle G^{\theta}(\beta, \mathbf{0} ; 0, \mathbf{0}) G^{\theta \dagger}(\beta, \mathbf{0} ; 0, \mathbf{0})\right\rangle}{\left\{\frac{1}{2 N_{\mathrm{c}}} \operatorname{Re} \operatorname{Tr}\left\langle G^{\theta}(\beta, \mathbf{0} ; 0, \mathbf{0})\right\rangle\right\}^{2}} .
$$

The factor $2 N_{\mathrm{c}}$ corresponds to the dimension of the propagator matrices.

The following physical interpretation can be suggested. Effectively we are computing the thermal expectation value of the imaginary part of the 4-quark operator, cf. eq. (3.14). But the contractions are non-trivial in that a quark-antiquark pair is generated at time 0 and annihilated at time $\beta$. The circling of the imaginary-time direction guarantees that the corresponding physical states are thermalized. The system is allowed to decide whether the propagation takes place in the form of open or bound states, as long as they are distributed according to the proper thermal weights. This physics is normalized by the propagators of two independent heavy quarks or antiquarks.

In order to write down the corresponding correlator for the octet case, eq. (2.9), we define the ratios

$$
\begin{aligned}
P_{1} & \equiv \frac{1}{2 N_{\mathrm{c}}} \operatorname{Re}\left\langle G_{\alpha \alpha ; i i}^{\theta}(\beta, \mathbf{0} ; 0, \mathbf{0})\right\rangle, \\
P_{2} & \equiv \frac{1}{2 N_{\mathrm{c}}}\left\langle G_{\alpha \gamma ; i j}^{\theta}(\beta, \mathbf{0} ; 0, \mathbf{0}) G_{\gamma \alpha ; j i}^{\theta \dagger}(\beta, \mathbf{0} ; 0, \mathbf{0})\right\rangle, \\
P_{3} & \equiv \frac{1}{2 N_{\mathrm{c}}^{2}}\left\langle G_{\alpha \alpha ; i j}^{\theta}(\beta, \mathbf{0} ; 0, \mathbf{0}) G_{\gamma \gamma ; j i}^{\theta \dagger}(\beta, \mathbf{0} ; 0, \mathbf{0})\right\rangle .
\end{aligned}
$$

Summing over the generators $T^{a}$ and normalizing the result according to eq. (2.19) we get

$$
\bar{S}_{8}=\frac{N_{\mathrm{c}}^{2} P_{3}-P_{2}}{\left(N_{\mathrm{c}}^{2}-1\right) P_{1}^{2}} .
$$

With the same notation, $\bar{S}_{1}=P_{2} / P_{1}^{2}$. Note that all the quantities entering the singlet and octet factors are manifestly gauge-invariant.

\section{Perturbative estimates}

In order to evaluate eq. (3.17) within (resummed) perturbation theory, we rewrite it in the form of a spectral representation. Denoting by $\rho_{q \bar{q}}(E, k)$ the spectral density of quarkantiquark states, and representing the spectral density of the single-particle states in the 


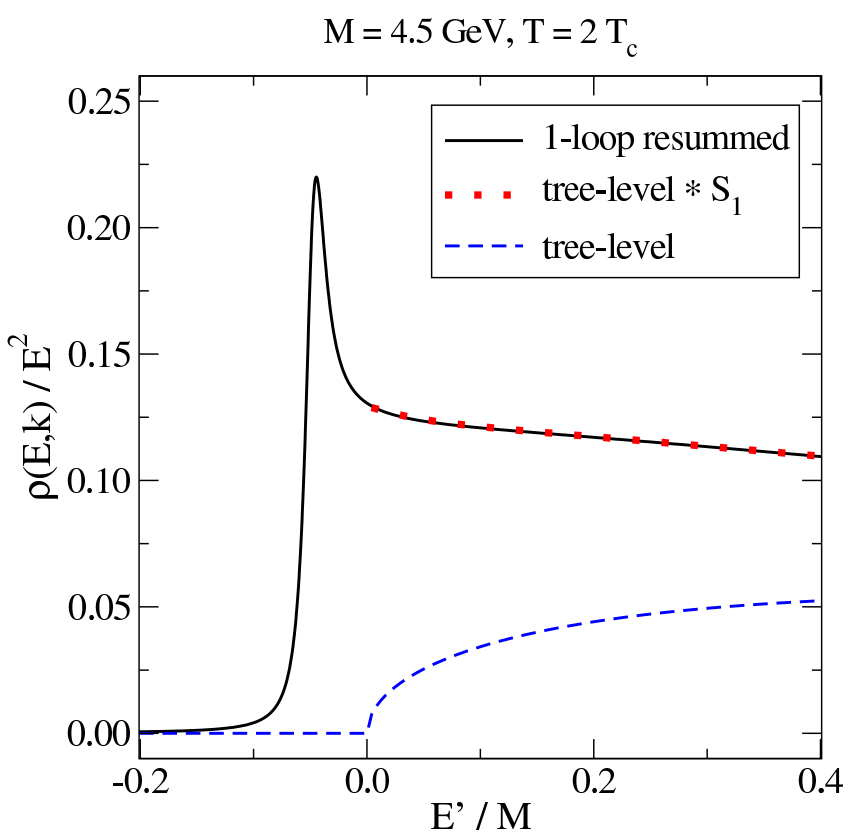

Figure 2. The tree-level (cf. eq. (4.3)) and 1-loop resummed (cf. refs. [26, 27]) pseudoscalar spectral function in hot QCD, with $E^{\prime} \equiv E-2 M-k^{2} /(4 M)$ denoting the energy with respect to the 2-particle threshold. Here $k=|\mathbf{k}|$ is the spatial momentum with respect to the heat bath. The tree-level result multiplied by the Sommerfeld factor $S_{1}$ from eq. (4.6) reproduces the abovethreshold spectral shape, but it misses the (thermally broadened) resonance-like contribution below the threshold.

denominator by the non-relativistic free form $\rho_{q}(E, p)=2 N_{\mathrm{c}} \pi \delta\left(E-E_{p}\right)$, the spectral representation of eq. (3.17) reads $^{4}$

$$
\bar{S}_{1} \approx \frac{\frac{1}{2 N_{\mathrm{c}}} \int_{\Lambda}^{\infty} \frac{\mathrm{d} E}{\pi} e^{-\beta E} \int_{\mathbf{k}} \rho_{q \bar{q}}(E, k)}{\int_{\mathbf{p}, \mathbf{q}} e^{-\beta\left(E_{p}+E_{q}\right)}} .
$$

Here $\mathbf{k}$ is the momentum of the quark-antiquark pair with respect to the heat bath. The Boltzmann weight $e^{-\beta E}$ implies that states with a small mass appear with a high likelihood.

We evaluate eq. (4.1) in three ways, with increasing sophistication. At tree-level, the spectral function originates from free scattering states, and reads

$$
\rho_{q \bar{q}}(E, k) \rightarrow \rho_{\text {tree }}(E, k) \equiv 2 N_{\mathrm{c}} \int_{\mathbf{p}, \mathbf{q}} \pi \delta\left(E-E_{p}-E_{q}\right)(2 \pi)^{3} \delta^{(3)}(\mathbf{k}-\mathbf{p}-\mathbf{q}) .
$$

Inserting this into eq. (4.1) and carrying out the integrals over $E$ and $\mathbf{k}$, immediately yields $\bar{S}_{1}=1$. For future reference, it is helpful to express the tree-level result in another way as well. Making use of the non-relativistic form $E_{p}=M+p^{2} /(2 M)$, the integrals over $\mathbf{p}, \mathbf{q}$ in eq. (4.2) are readily carried out, yielding

$$
\rho_{\text {tree }}(E, k)=\frac{N_{\mathrm{c}} M^{\frac{3}{2}} \theta\left(E^{\prime}\right) \sqrt{E^{\prime}}}{2 \pi}, \quad E^{\prime} \equiv E-2 M-\frac{k^{2}}{4 M} .
$$

\footnotetext{
${ }^{4}$ The numerator is analogous to the relativistic form in eq. (3.1); the non-relativistic contribution originates from the first term. The peculiar nature of the Wick contractions discussed in section 3.3, related to the non-relativistic propagators used, subsequently sets $\tau \rightarrow \beta$.
} 
This result is illustrated with a dashed line in figure 2. We note that it amounts to $-1 / 3$ times the vector channel spectral function studied in ref. [26], i.e. the pseudoscalar channel [27].

As a second step, we include the Sommerfeld enhancement in its standard form. Employing QCD language, we refer to this approximation as a contribution from "open" (or "above-threshold", or "scattering") states. Then

$$
\rho_{q \bar{q}}(E, k) \rightarrow \rho_{\text {open }}(E, k) \equiv 2 N_{\mathrm{c}} \int_{\mathbf{p}, \mathbf{q}} \pi \delta\left(E-E_{p}-E_{q}\right)(2 \pi)^{3} \delta^{(3)}(\mathbf{k}-\mathbf{p}-\mathbf{q}) S_{1}(v),
$$

where $v=|\mathbf{v}|$ is the relative velocity defined through

$$
\mathbf{v} \equiv \frac{\mathbf{p}-\mathbf{q}}{2 M}
$$

The Sommerfeld factor reads [3]

$$
S_{1}=\frac{X_{1}}{1-e^{-X_{1}}}, \quad X_{1}=\frac{g^{2} C_{\mathrm{F}}}{4 v} .
$$

Eqs. (4.4) and (4.5) imply that $E_{p}+E_{q}=2 M+k^{2} /(4 M)+M v^{2}$, so we can identify the variable $E^{\prime}$ of eq. (4.3) as $E^{\prime}=M v^{2}$. Therefore $\rho_{\text {open }}$ can be evaluated by multiplying $\rho_{\text {tree }}$ from eq. (4.3) by $S_{1}(v)$; the result is shown with a dotted line in figure 2 .

We note in passing that expressing both the numerator and denominator of eq. (4.1) in center-of-mass variables, and inserting $\rho_{\text {open }}$ from eq. (4.4), a simple computation yields

$$
\bar{S}_{1, \text { open }}=\frac{\int_{\mathbf{v}} e^{-\frac{M v^{2}}{T}} S_{1}(v)}{\int_{\mathbf{v}} e^{-\frac{M v^{2}}{T}}} .
$$

This agrees with the expression given in eq. (2.15).

As a third level of sophistication, we evaluate eq. (4.1) by including the full spectral shape shown in figure 2. We refer to this as a contribution from "open+bound" states. Substituting variables as $E^{\prime}=E-2 M-k^{2} /(4 M)$; noting that the 1-loop resummed spectral function ${ }^{5}$ depends on $E^{\prime}$ only [26], so that we can write $\rho_{q \bar{q}}(E, k)=\rho\left(E^{\prime}\right)$; and carrying out both integrals in the denominator of eq. (4.1) as well as the integral over $\mathbf{k}$ in the numerator, yields

$$
\bar{S}_{1, \text { open+bound }}=\frac{1}{2 N_{\mathrm{c}}}\left(\frac{4 \pi}{M T}\right)^{\frac{3}{2}} \int_{-\Lambda^{\prime}}^{\infty} \frac{\mathrm{d} E^{\prime}}{\pi} e^{-\frac{E^{\prime}}{T}} \rho\left(E^{\prime}\right) .
$$

The cutoff scale $\Lambda^{\prime}$ has no practical significance, given that the spectral function vanishes for $E^{\prime} \ll-\alpha_{\mathrm{S}}^{2} M$ (cf. figure 2).

\footnotetext{
${ }^{5}$ The 1-loop resummed spectral function corresponds to the imaginary part of a Coulomb Green's function, with the potential appearing in the inhomogoneous Schrödinger equation computed up to order $g^{2}$ in Hard Thermal Loop resummed perturbation theory. This potential has a real part, accounting for "virtual" thermal corrections such as Debye screening, as well as an imaginary part, accounting for "real" thermal corrections such as elastic $2 \rightarrow 2$ scatterings with light plasma constituents, which lead to thermal broadening.
} 

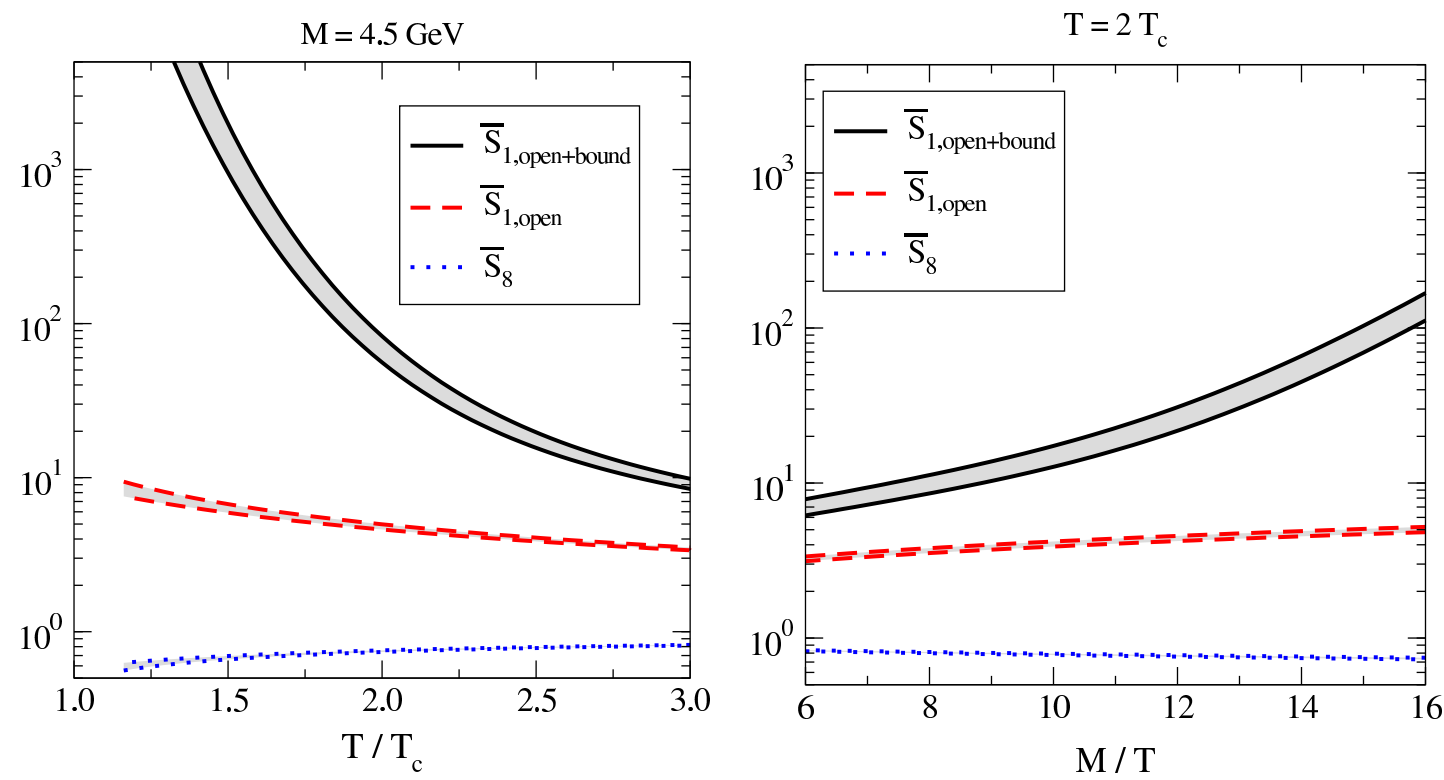

Figure 3. Perturbative Sommerfeld factors, as a function of $T$ for fixed $M$ (left), and as a function of $M$ for fixed $T$ (right). As a gauge coupling we have used the 2-loop dimensionally reduced coupling from ref. [28], with $\Lambda_{\overline{\mathrm{MS}}} \simeq 360 \mathrm{MeV}$ [29], and moreover we chose $T_{\mathrm{c}} \simeq 155 \mathrm{MeV}$ (for a review, see ref. [30]). The numerical values vary in the range $\alpha_{\mathrm{s}} \simeq 0.2 \ldots 0.4$. The bands follow from variations of the renormalization scale by a factor $1 / 2 \ldots 2$ around a typical thermal choice $\sim 2 \pi T$, but represent only a lower bound of theoretical uncertainties.

A numerical evaluation of eq. (4.8), based on the spectral function shown in figure 2, is illustrated in figure 3. It is obvious that at low temperatures, the bound state contribution completely dominates the result, boosting $\bar{S}_{1}$ by more than two orders of magnitude.

In the octet case, where the interaction is repulsive, we only include the contribution from the open states, like in eq. (4.7). The octet Sommerfeld factor reads [3]

$$
S_{8}=\frac{X_{8}}{e^{X_{8}-1}}, \quad X_{8}=\left(\frac{N_{\mathrm{c}}}{2}-C_{\mathrm{F}}\right) \frac{g^{2}}{4 v} .
$$

Numerical results are displayed in figure 3, showing a modest suppression.

\section{Lattice analysis}

We have measured the observables defined in eqs. (3.17)-(3.21) using lattice NRQCD. The gauge configuration ensemble corresponds to $N_{\mathrm{f}}=2+1$ dynamical quark flavors at a temperature of a few hundred $\mathrm{MeV}$. In this exploratory study, a single lattice spacing and a single spatial volume were employed. The same thermal gauge configurations have previously been used for studying in-medium modifications of bottomonium spectral functions [31] and the electric conductivity of the quark-gluon plasma [32].

The configurations used correspond to an anisotropic lattice setup, with the spatial lattice spacing $a_{s}$ and the temporal one $a_{t}$ related through $a_{s} / a_{t} \approx 3.5$. The tuning of the anisotropy parameter and the vacuum properties of the lattice action were studied by the Hadron Spectrum Collaboration [33, 34], yielding $a_{s}=0.1227(8) \mathrm{fm}, M_{\pi} \simeq 400 \mathrm{MeV}$, 


\begin{tabular}{|lllllllll|}
\hline$N_{\tau}$ & $T / T_{\mathrm{c}}$ & $a_{s} M_{\text {kin }}$ & $M_{\text {kin }} / T$ & $10^{4} P_{1}$ & $10^{5} P_{2}$ & $10^{6} P_{3}$ & $\bar{S}_{1}$ & $\bar{S}_{8}$ \\
\hline 32 & 0.95 & 2.92 & 26.7 & $0.75(7)$ & $0.938(5)$ & $1.06(1)$ & $1690(410)$ & $3.6(15)$ \\
28 & 1.09 & 2.92 & 23.4 & $3.0(1)$ & $2.78(1)$ & $3.19(3)$ & $301(35)$ & $1.2(3)$ \\
24 & 1.27 & 2.92 & 20.0 & $8.0(3)$ & $8.64(3)$ & $10.3(1)$ & $134(13)$ & $1.2(2)$ \\
20 & 1.52 & 2.92 & 16.7 & $24.4(5)$ & $28.5(1)$ & $36.1(4)$ & $48(3)$ & $0.83(6)$ \\
16 & 1.90 & 2.92 & 13.3 & $68.9(9)$ & $102.2(3)$ & $147(1)$ & $21.6(6)$ & $0.80(2)$ \\
\hline 32 & 0.95 & 1.50 & 13.7 & $1.31(7)$ & $1.308(8)$ & $1.51(1)$ & $758(128)$ & $3.7(5)$ \\
28 & 1.09 & 1.50 & 12.0 & $4.6(1)$ & $3.04(2)$ & $3.62(3)$ & $142(15)$ & $1.25(9)$ \\
24 & 1.27 & 1.50 & 10.3 & $10.4(2)$ & $7.38(4)$ & $9.21(8)$ & $69(5)$ & $1.05(5)$ \\
20 & 1.52 & 1.50 & 8.57 & $25.7(3)$ & $19.7(1)$ & $27.2(2)$ & $30(2)$ & $0.89(3)$ \\
16 & 1.90 & 1.50 & 6.86 & $61.9(6)$ & $60.1(3)$ & $95.8(8)$ & $15.7(3)$ & $0.85(1)$ \\
\hline 16 & 1.90 & 2.00 & 9.14 & $57.6(6)$ & $55.7(2)$ & $86.4(8)$ & $16.8(4)$ & $0.83(1)$ \\
16 & 1.90 & 2.50 & 11.4 & $62.5(7)$ & $76.0(2)$ & $113(1)$ & $19.5(5)$ & $0.81(2)$ \\
\hline
\end{tabular}

Table 1. Parameters and results of the unquenched $N_{\mathrm{f}}=2+1$ flavour simulations, with $N_{\tau}$ denoting the number of lattice points in the time direction. The 4-volume in lattice units was fixed at $N_{\tau} \times 24^{3}$; the lattice spacings are asymmetric, with $a_{s} / a_{\tau} \approx 3.5$. The same configurations $\left(10^{3}\right.$ per parameter set) were previously used e.g. in refs. [31, 32]. Errors are statistical only; for $\bar{S}_{1}$ and $\bar{S}_{8}$ they were obtained with a jackknife analysis. The results of this table are illustrated in figure 4 .

$M_{K} \simeq 500 \mathrm{MeV}$. The critical temperature was estimated from the behaviour of the Polyakov loop expectation value [35], with the result $T_{\mathrm{c}}=185(4) \mathrm{MeV}$. The heavy quark mass for the physical bottom case, $a_{s} M_{\text {kin }}=2.92$, was tuned by extracting a kinetic mass from dispersion relations and matching it onto the spin-averaged mass of the $\eta_{b}$ and $\Upsilon$ mesons. Further details on the lattice setup can be found in ref. [31].

It is important to note that the contribution of the bare heavy quark "rest mass" drops out in the ratios in eqs. (3.17) and (3.21), and radiatively generated self-energy divergences drop out as well. Therefore we can leave out the bare rest mass from the equation of motion defining the heavy quark propagator, which facilitates the measurement. The form of the NRQCD propagator is specified in appendix A.

The main results of our lattice study are given in table 1 and in figure 4 . We note that in addition to our main $N_{\mathrm{f}}=2+1$ lattice calculation, we have also carried out tests on quenched lattice gauge configurations with a symmetric lattice spacing on a small lattice volume $4 \times 8^{3}$. For completeness the result of quenched test is shown in table 2 .

Comparing our $N_{\mathrm{f}}=2+1$ lattice results in figure 4 with the perturbative results in figure 3 , the following conclusions can be drawn:

(i) The lattice and perturbative results for $\bar{S}_{8}$ are of similar magnitudes, and at $T \gtrsim 1.5 T_{\mathrm{c}}$ both are somewhat below unity. At smaller $T / T_{\mathrm{c}}$ the central values of the lattice results are larger, however the observable in eq. (3.21) involves a subtraction in the numerator and thus a large cancellation at $T \lesssim 1.5 T_{\mathrm{c}}$. This is likely to lead 


\begin{tabular}{|lllllllll|}
\hline$\beta_{0}$ & $T / T_{\mathrm{c}}$ & $a_{s} M_{\text {kin }}$ & $M_{\text {kin }} / T$ & $10^{2} P_{1}$ & $10^{3} P_{2}$ & $10^{4} P_{3}$ & $\bar{S}_{1}$ & $\bar{S}_{8}$ \\
\hline 5.85 & 1.33 & 3.289 & 13.2 & $2.5(1)$ & $6.26(4)$ & $11.4(5)$ & $10.3(7)$ & $0.81(7)$ \\
5.90 & 1.47 & 2.988 & 12.0 & $2.06(8)$ & $4.19(3)$ & $7.9(3)$ & $9.9(6)$ & $0.86(5)$ \\
5.95 & 1.62 & 2.724 & 10.9 & $2.17(6)$ & $3.07(2)$ & $6.9(3)$ & $6.6(2)$ & $0.83(1)$ \\
6.00 & 1.77 & 2.498 & 10.0 & $2.01(5)$ & $2.20(1)$ & $5.6(2)$ & $5.4(3)$ & $0.86(2)$ \\
6.05 & 1.93 & 2.302 & 9.21 & $1.81(4)$ & $1.64(1)$ & $4.2(1)$ & $5.0(2)$ & $0.83(2)$ \\
\hline
\end{tabular}

Table 2. Parameters and results of the quenched simulations, with $\beta_{0}$ denoting the coefficient of the Wilson plaquette term. The 4 -volume in lattice units was fixed at $4 \times 8^{3}$. Conversions to units of $T_{\mathrm{c}}$ are based on ref. [36]. We show these small-scale isotropic $\left(a_{s} / a_{\tau}=1\right)$ lattice results (from 200 gauge configurations) in order to permit for a rapid crosscheck of the measurement algorithm.
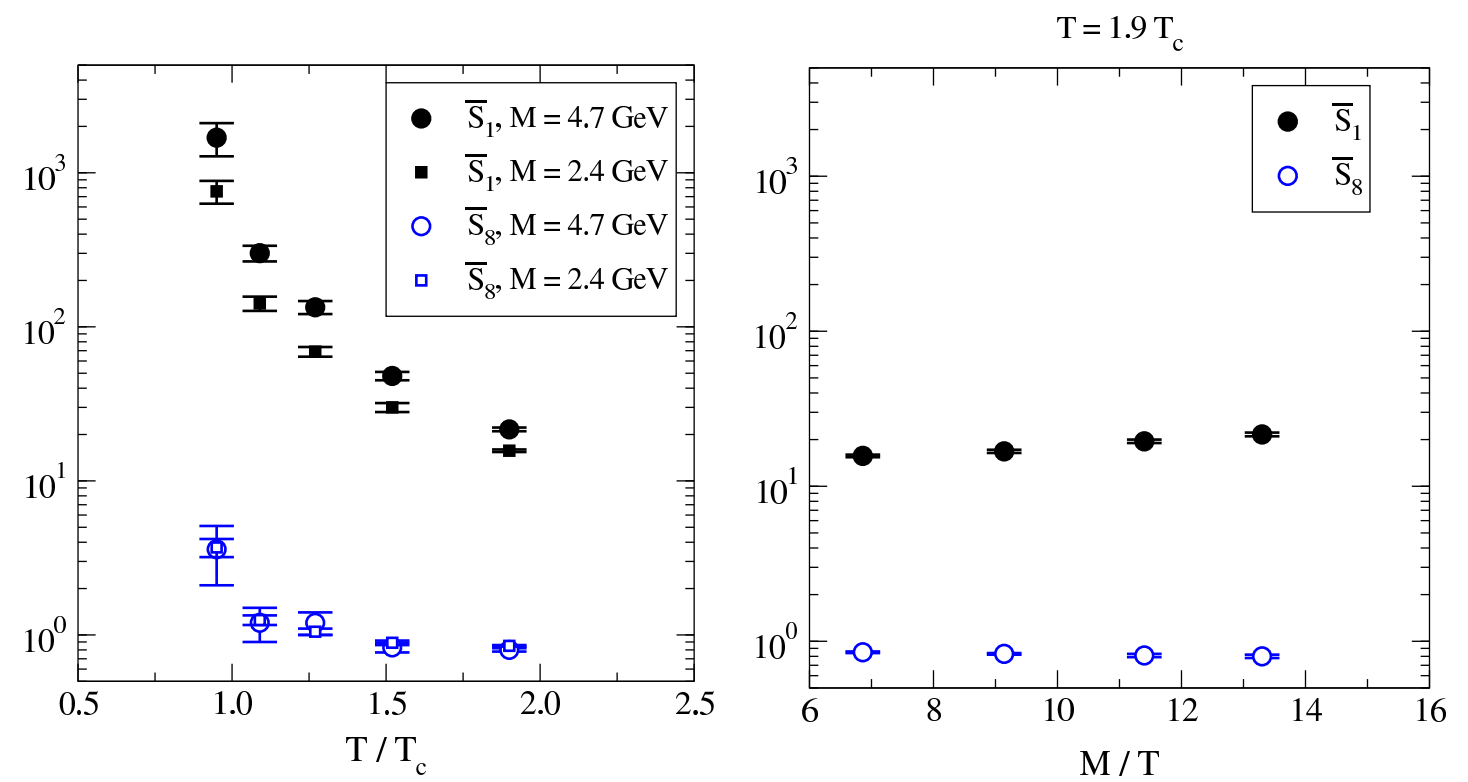

Figure 4. Lattice NRQCD estimates of the thermal Sommerfeld factors. Left: results for fixed $M$ as a function of $T / T_{\mathrm{c}}$, with $T_{\mathrm{c}} \simeq 185 \mathrm{MeV}$. Right: results for a fixed $T / T_{\mathrm{c}} \approx 1.9$ as a function of $M / T$. The parameters cannot be chosen exactly the same as in figure 3 because the lattice ensemble corresponds to an unphysical value of $M_{\pi}$ and does not represent an infinite-volume and continuum extrapolation. Nevertheless a reasonable qualitative agreement with figure 3 can be observed.

to systematic uncertainties from lattice artifacts which may be much larger than the statistical ones.

(ii) The lattice results for $\bar{S}_{1}$ can exceed $10^{3}$, whereas the perturbative ones according to the prescription used in the literature, which we have denoted by $\bar{S}_{1, \text { open }}$ (cf. eq. (4.7)), never exceed 10. Even though the perturbative result may be expected to have $\sim 50 \%$ uncertainties (see below), such a discrepancy of two orders of magnitude cannot be accounted for by conceivable perturbative uncertainties.

(iii) The lattice results for $\bar{S}_{1}$ are in qualitative agreement with the perturbative ones including bound state contributions, which we have denoted by $\bar{S}_{1, \text { open+bound }}$. Cer- 
tain differences do remain: the mass dependence at fixed $T$ is more rapid in the perturbative results (cf. right panels of figures 3 and 4), and the temperature scales are somewhat shifted (cf. left panels of figures 3 and 4). We stress, however, that the vertical axes of the plots are logarithmic; without the inclusion of bound state contributions, there would be differences of up to two orders of magnitude. With the inclusion of bound states, orders of magnitude can be accounted for. For the remaining discrepancies there are many likely explanations. First of all our leading-order perturbative computation has uncertainties on the $\sim 50 \%$ level. Amongst others, the perturbative predictions are sensitive to the parameter values used, in particular the scale chosen for the gauge coupling and the scheme chosen for the quark mass; in the absence of an actual higher-order computation of $\bar{S}_{1}$, the choices made amount to ad hoc recipes. Second, the lattice results correspond to quark masses larger than in the continuum computation, which implies that physical scales cannot be compared unambiguously (this is reflected by the different values of $T_{\mathrm{c}} / \mathrm{MeV}$ ). Third, the lattice results have no continuum limit and take place in a finite volume, $V \approx(2.9 \mathrm{fm})^{3}$. It would be interesting to carry out a refined lattice analysis and a systematic higher-order perturbative computation in order to see if the discrepancies get thereby reduced.

\section{Discussion and outlook}

We have presented a power-counting argument showing that bound states (if they exist) dominate the thermal co-annihilation rate of kinetically equilibrated non-relativistic particles at low temperatures (discussion around eq. (2.3)), as well as a theoretical derivation of basic formulae (eqs. (3.14), (3.17), (3.21) and (4.8)) which permit for the inclusion of bound state contributions in perturbative (section 4 ) or non-perturbative (section 5) studies. The perturbative side can be reduced to the determination of a spectral function, i.e. the imaginary part of a particular Green's function, with a thermal potential incorporating the effects of Debye screening and soft $2 \rightarrow 2$ scatterings of the co-annihilating particles with light plasma constituents. The results of both perturbative and non-perturbative computations can be parametrized by generalizations of the so-called thermal Sommerfeld factors.

Even though our study was inspired by the co-annihilation rate of weakly interacting non-relativistic particles in cosmology, which determines their freeze-out temperature, we applied the formalism to obtain a non-perturbative estimate of the heavy quark chemical equilibration rate in QCD, relevant for heavy ion collision experiments. Our numerical investigation confirmed the existence of Sommerfeld enhancement in the case of attractive interactions as well as a reduction (at $T \gtrsim 1.5 T_{\mathrm{c}}$ ) in the case of repulsive interactions (cf. figure 4 ). At $T \gtrsim 1.5 T_{\mathrm{c}}$ the repulsive case shows reasonable agreement with perturbation theory (cf. figure 3 ).

In the attractive case, corresponding to the factor $\bar{S}_{1}$, we found a much larger Sommerfeld enhancement than predicted by the perturbative formula sometimes used in literature (cf. eq. (4.7)). In accordance with the power-counting argument around eq. (2.3) we believe that the difference originates from bound state contributions, which were omitted in 
eq. (4.7). ${ }^{6}$ Due to their smaller mass, bound states appear with a less suppressed Boltzmann weight in the thermal ensemble than scattering states. Note also that bound state formation is a relatively speaking fast process, without any exponential suppression factors; thermal friction, caused by $2 \rightarrow 2$ scatterings with light plasma constituents, lets an open $q \bar{q}$ pair lose energy until it appears with its proper thermal weight. Including bound state decays through a numerical determination of the imaginary part of a thermal Green's function, $\rho_{q \bar{q}}(E, k)$, we did find qualitative agreement between perturbative and lattice results (cf. figures 3 and 4 ).

It may be asked to what extent our findings could be relevant for the weakly interacting cases considered in cosmology. First of all we note that typical parameter values playing a role in cosmology are $\alpha_{\mathrm{w}} \sqrt{M / T} \sim 0.04 \sqrt{20} \sim 0.2$, whereas we have simulated $\alpha_{\mathrm{s}} \sqrt{M / T} \sim$ $0.25 \sqrt{(2.4 \ldots 4.7) /(0.2 \ldots 0.4)} \sim 0.6 \ldots 1.2$. This means that we have been deeper in the enhancement regime than is typical for cosmology. That said, there are cosmological models in which bound state formation has been demonstrated in vacuum (cf. e.g. refs. [37-40]). It might be interesting to use our formalism to study whether or not bound states could affect the thermal freeze-out process in these cases. Another direction worth a look are models based on strongly interacting dark sectors (cf. e.g. refs. [41-44] and references therein).

Let us finally return from cosmology to hot QCD. Inserting the values of the absorptive parts of the 4-quark coefficients from ref. [15] and assuming that the octet Sommerfeld factors are spin-independent, the heavy quark chemical equilibration rate evaluates to [13]

$$
\Gamma_{\text {chem }} \simeq \frac{8 \pi \alpha_{\mathrm{s}}^{2}}{3 M^{2}}\left(\frac{M T}{2 \pi}\right)^{3 / 2} e^{-M / T}\left[\frac{\bar{S}_{1}}{3}+\left(\frac{5}{6}+N_{\mathrm{f}}\right) \bar{S}_{8}\right]
$$

Inserting the highest temperature reached in current heavy-ion collisions, $T \sim 400 \mathrm{MeV}$, a charm quark mass $M \sim 1.5 \mathrm{GeV}$, a running coupling $\alpha_{\mathrm{s}} \sim 0.25$, and values $\bar{S}_{1} \sim 15$, $\bar{S}_{8} \sim 0.8$ as estimated in the current study for $N_{\mathrm{f}}=3$, we get $\Gamma_{\text {chem }}^{-1} \sim 150 \mathrm{fm} /$ c. Therefore heavy quark chemical equilibration is unlikely to take place within the lifetime $\sim 10 \mathrm{fm} / \mathrm{c}$ of the current generation of heavy ion collision experiments. This can be compared with their kinetic equilibration time scale which could be as small as $\sim 1 \mathrm{fm} / \mathrm{c}$ in the case of charm quarks $[45,46]$. It may be noted, however, that $\Gamma_{\text {chem }}$ (eq. (6.1)) changes rapidly with temperature. Already a modest increase, in the ballpark of the planned Future Circular Collider (FCC) heavy ion program [47], could therefore yield chemically equilibrated charm quarks.

\section{Acknowledgments}

We thank the FASTSUM collaboration for providing the gauge configurations used in our unquenched measurements. M.L. thanks D. Bödeker for collaboration at initial stages of this project, and M. Garny for a helpful discussion. S.K. thanks AEC of the University of Bern for hospitality and support during the completion of this work. S.K. was supported

\footnotetext{
${ }^{6}$ Bound state contributions have been included in thermal QCD literature in other contexts, for instance in studies of the leptonic decays of heavy quark-antiquark pairs. However these processes are too slow by many orders of magnitude to contribute to heavy quark chemical equilibration.
} 
by the National Research Foundation of Korea under grant No. 2015R1A2A2A01005916 funded by the Korean government (MEST). M.L. was supported by the Swiss National Science Foundation (SNF) under grant 200020-155935.

\section{A Heavy quark propagators}

For completeness we reiterate here the non-perturbative definitions of heavy quark and antiquark propagators within the NRQCD framework. Denoting

$$
\begin{aligned}
& G_{\alpha \gamma ; i j}^{\theta}\left(\tau_{2}, \mathbf{x} ; \tau_{1}, \mathbf{y}\right) \equiv\left\langle\theta_{\alpha i}\left(\tau_{2}, \mathbf{x}\right) \theta_{\gamma j}^{*}\left(\tau_{1}, \mathbf{y}\right)\right\rangle \\
& G_{\alpha \gamma ; i j}^{\chi}\left(\tau_{2}, \mathbf{x} ; \tau_{1}, \mathbf{y}\right) \equiv\left\langle\chi_{\alpha i}\left(\tau_{2}, \mathbf{x}\right) \chi_{\gamma j}^{*}\left(\tau_{1}, \mathbf{y}\right)\right\rangle
\end{aligned}
$$

the fields "naturally" propagate into the regions $\tau_{2}>\tau_{1}$ and $\tau_{1}>\tau_{2}$, respectively, given that $\theta^{\dagger}$ and $\chi$ correspond to creation operators for quarks and antiquarks. Specifically, in continuum notation and in a given gauge field background,

$$
\left(D_{\tau_{2}}+M_{\text {rest }}-\frac{\vec{D}^{2}}{2 M_{\text {kin }}}+\ldots\right) G^{\theta}\left(\tau_{2}, \mathbf{x} ; \tau_{1}, \mathbf{y}\right)=0, \quad \tau_{2}>\tau_{1}
$$

with the initial condition

$$
G^{\theta}\left(\tau_{1}^{+}, \mathbf{x} ; \tau_{1}, \mathbf{y}\right)=\mathbb{1}_{2 N_{\mathrm{c}} \times 2 N_{\mathrm{c}}} \delta^{(3)}(\mathbf{x}-\mathbf{y}) .
$$

The boundary condition

$$
G^{\theta}\left(0, \mathbf{x} ; \tau_{1}, \mathbf{y}\right) \equiv-G^{\theta}\left(\beta, \mathbf{x} ; \tau_{1}, \mathbf{y}\right)
$$

allows us to go into the region $\tau_{2}<\tau_{1}$ (cf. figure 1 ). The propagator for $\chi$ is obtained from

$$
G^{\chi}\left(\tau_{2}, \mathbf{x} ; \tau_{1}, \mathbf{y}\right)=-\left[G^{\theta}\left(\tau_{1}, \mathbf{y} ; \tau_{2}, \mathbf{x}\right)\right]^{\dagger} .
$$

We stress that, as mentioned in the text, the contribution of the rest mass, $M_{\text {rest }}$, appearing linearly in eq. (A.3), drops out in our final observables, eqs. (3.17) and (3.21). Therefore this term can be omitted from the practical computations.

On the lattice the non-relativistic quark propagator is calculated from $(\cdot \equiv 0, \mathbf{0})$

$$
\begin{aligned}
G^{\theta}(0, \mathbf{x} ; \cdot) & =\frac{\delta_{\mathbf{x}, \mathbf{0}}}{a_{s}^{3}} \\
G^{\theta}\left(a_{t}, \mathbf{x} ; \cdot\right) & =\left(1-\frac{a_{t} \mathcal{H}_{0}}{2 n}\right)^{n} U_{0}^{\dagger}(0, \mathbf{x})\left(1-\frac{a_{t} \mathcal{H}_{0}}{2 n}\right)^{n} G^{\theta}(0, \mathbf{x} ; \cdot), \\
G^{\theta}\left(\tau+a_{t}, \mathbf{x} ; \cdot\right) & =\left(1-\frac{a_{t} \mathcal{H}_{0}}{2 n}\right)^{n} U_{0}^{\dagger}(\tau, \mathbf{x})\left(1-\frac{a_{t} \mathcal{H}_{0}}{2 n}\right)^{n}\left(1-a_{t} \delta \mathcal{H}\right) G^{\theta}(\tau, \mathbf{x} ; \cdot),
\end{aligned}
$$

where $U_{0}$ is a time-direction gauge link. The lowest-order Hamiltonian reads

$$
\mathcal{H}_{0}=-\frac{\Delta^{(2)}}{2 M_{\text {kin }}},
$$


where $\Delta^{(2)}$ is a discretized gauge Laplacian. The higher order correction is

$$
\begin{aligned}
\delta \mathcal{H}= & -\frac{\left(\Delta^{(2)}\right)^{2}}{8 M_{\mathrm{kin}}^{3}}+\frac{i g_{0}(\nabla \cdot \mathbf{E}-\mathbf{E} \cdot \nabla)}{8 M_{\mathrm{kin}}^{2}}-\frac{g_{0} \sigma \cdot(\nabla \times \mathbf{E}-\mathbf{E} \times \nabla)}{8 M_{\mathrm{kin}}^{2}} \\
& -\frac{g_{0} \sigma \cdot \mathbf{B}}{2 M_{\mathrm{kin}}}+\frac{a_{s}^{2} \Delta^{(4)}}{24 M_{\mathrm{kin}}}-\frac{a_{t}\left(\Delta^{(2)}\right)^{2}}{16 n M_{\mathrm{kin}}^{2}},
\end{aligned}
$$

where $g_{0}$ is the bare gauge coupling, and unspecified notation is explained in ref. [31]. The parameter $n$, which stabilizes the high-momentum behaviour of the propagator, is set to 1 for $a_{s} M_{\text {kin }}=2.92$ and to 3 for $a_{s} M_{\text {kin }}=1.5$. The electric and magnetic fields appearing in eq. (A.11) were implemented in a tadpole-improved [48] form, with the improvement factor $u_{s}=0.7336$ for the spatial link and $u_{\tau}=1.0$ for the time link [33].

Open Access. This article is distributed under the terms of the Creative Commons Attribution License (CC-BY 4.0), which permits any use, distribution and reproduction in any medium, provided the original author(s) and source are credited.

\section{References}

[1] A. Sommerfeld, Über die Beugung und Bremsung der Elektronen, Ann. Phys. (Leipzig) 403 (1931) 257.

[2] L.D. Landau and E.M. Lifshitz, Quantum mechanics, non-relativistic theory, $3^{\text {rd }}$ edition, Butterworth-Heinemann, Oxford U.K. (1977), see $\S 136$.

[3] V.S. Fadin, V.A. Khoze and T. Sjöstrand, On the threshold behavior of heavy top production, Z. Phys. C 48 (1990) 613 [inSPIRE].

[4] J. Hisano, S. Matsumoto, M. Nagai, O. Saito and M. Senami, Non-perturbative effect on thermal relic abundance of dark matter, Phys. Lett. B 646 (2007) 34 [hep-ph/0610249] [INSPIRE].

[5] M. Cirelli, A. Strumia and M. Tamburini, Cosmology and astrophysics of minimal dark matter, Nucl. Phys. B 787 (2007) 152 [arXiv:0706.4071] [InSPIRE].

[6] J.L. Feng, M. Kaplinghat and H.-B. Yu, Sommerfeld enhancements for thermal relic dark matter, Phys. Rev. D 82 (2010) 083525 [arXiv:1005.4678] [INSPIRE].

[7] A. Hryczuk, R. Iengo and P. Ullio, Relic densities including Sommerfeld enhancements in the MSSM, JHEP 03 (2011) 069 [arXiv:1010.2172] [INSPIRE].

[8] M. Beneke, C. Hellmann and P. Ruiz-Femenia, Non-relativistic pair annihilation of nearly mass degenerate neutralinos and charginos III. Computation of the Sommerfeld enhancements, JHEP 05 (2015) 115 [arXiv: 1411.6924] [INSPIRE].

[9] T. Biró and J. Zimányi, Quarkochemistry in relativistic heavy ion collisions, Phys. Lett. B 113 (1982) 6 [inSPIRE].

[10] J. Rafelski and B. Müller, Strangeness production in the quark-gluon plasma, Phys. Rev. Lett. 48 (1982) 1066 [Erratum ibid. 56 (1986) 2334] [INSPIRE].

[11] T. Matsui, B. Svetitsky and L.D. McLerran, Strangeness production in ultrarelativistic heavy ion collisions. 1. Chemical kinetics in the quark-gluon plasma, Phys. Rev. D 34 (1986) 783 [Erratum ibid. D 37 (1988) 844] [INSPIRE]. 
[12] D. Bödeker and M. Laine, Heavy quark chemical equilibration rate as a transport coefficient, JHEP 07 (2012) 130 [arXiv: 1205.4987] [INSPIRE].

[13] D. Bödeker and M. Laine, Sommerfeld effect in heavy quark chemical equilibration, JHEP 01 (2013) 037 [arXiv: 1210.6153] [INSPIRE].

[14] W.E. Caswell and G.P. Lepage, Effective lagrangians for bound state problems in QED, QCD and other field theories, Phys. Lett. B 167 (1986) 437 [INSPIRE].

[15] G.T. Bodwin, E. Braaten and G.P. Lepage, Rigorous QCD analysis of inclusive annihilation and production of heavy quarkonium, Phys. Rev. D 51 (1995) 1125 [Erratum ibid. D 55 (1997) 5853] [hep-ph/9407339] [INSPIRE].

[16] J. Bernstein, L.S. Brown and G. Feinberg, The cosmological heavy neutrino problem revisited, Phys. Rev. D 32 (1985) 3261 [INSPIRE].

[17] K. Griest and D. Seckel, Three exceptions in the calculation of relic abundances, Phys. Rev. D 43 (1991) 3191 [inSPIRE].

[18] P. Gondolo and G. Gelmini, Cosmic abundances of stable particles: Improved analysis, Nucl. Phys. B 360 (1991) 145 [inSPIRE].

[19] J.L. Feng, M. Kaplinghat, H. Tu and H.-B. Yu, Hidden charged dark matter, JCAP 07 (2009) 004 [arXiv: 0905. 3039] [INSPIRE].

[20] M.B. Wise and Y. Zhang, Stable bound states of asymmetric dark matter, Phys. Rev. D 90 (2014) 055030 [Erratum ibid. 91 (2015) 039907] [arXiv:1407.4121] [INSPIRE].

[21] B. von Harling and K. Petraki, Bound-state formation for thermal relic dark matter and unitarity, JCAP 12 (2014) 033 [arXiv:1407.7874] [INSPIRE].

[22] J. Ellis, F. Luo and K.A. Olive, Gluino coannihilation revisited, JHEP 09 (2015) 127 [arXiv: 1503.07142] [INSPIRE].

[23] K. Petraki, M. Postma and M. Wiechers, Dark-matter bound states from Feynman diagrams, JHEP 06 (2015) 128 [arXiv: 1505.00109] [INSPIRE].

[24] A. Petrelli, M. Cacciari, M. Greco, F. Maltoni and M.L. Mangano, NLO production and decay of quarkonium, Nucl. Phys. B 514 (1998) 245 [hep-ph/9707223] [INSPIRE].

[25] G.T. Bodwin and A. Petrelli, Order-v $v^{4}$ corrections to $S$-wave quarkonium decay, Phys. Rev. D 66 (2002) 094011 [Erratum ibid. D 87 (2013) 039902] [arXiv:1301.1079] [INSPIRE].

[26] M. Laine, A resummed perturbative estimate for the quarkonium spectral function in hot QCD, JHEP 05 (2007) 028 [arXiv:0704.1720] [INSPIRE].

[27] Y. Burnier, M. Laine and M. Vepsäläinen, Heavy quarkonium in any channel in resummed hot QCD, JHEP 01 (2008) 043 [arXiv:0711.1743] [INSPIRE].

[28] M. Laine and Y. Schröder, Two-loop QCD gauge coupling at high temperatures, JHEP 03 (2005) 067 [hep-ph/0503061] [INSPIRE].

[29] PACS-CS collaboration, S. Aoki et al., Precise determination of the strong coupling constant in $N_{f}=2+1$ lattice QCD with the Schrödinger functional scheme, JHEP 10 (2009) 053 [arXiv:0906.3906] [INSPIRE].

[30] H.B. Meyer, QCD at non-zero temperature from the lattice, PoS (LATTICE 2015) 014 [arXiv: 1512.06634] [INSPIRE]. 
[31] G. Aarts et al., The bottomonium spectrum at finite temperature from $N_{f}=2+1$ lattice QCD, JHEP 07 (2014) 097 [arXiv: 1402.6210] [INSPIRE].

[32] G. Aarts, C. Allton, A. Amato, P. Giudice, S. Hands and J.-I. Skullerud, Electrical conductivity and charge diffusion in thermal QCD from the lattice, JHEP 02 (2015) 186 [arXiv: 1412.6411] [INSPIRE].

[33] R.G. Edwards, B. Joo and H.-W. Lin, Tuning for three-flavors of anisotropic clover fermions with Stout-link smearing, Phys. Rev. D 78 (2008) 054501 [arXiv:0803.3960] [INSPIRE].

[34] Hadron Spectrum collaboration, H.-W. Lin et al., First results from $2+1$ dynamical quark flavors on an anisotropic lattice: light-hadron spectroscopy and setting the strange-quark mass, Phys. Rev. D 79 (2009) 034502 [arXiv:0810.3588] [INSPIRE].

[35] C. Allton et al., $2+1$ flavour thermal studies on an anisotropic lattice, PoS (LATTICE 2013) 151 [arXiv: 1401.2116] [INSPIRE].

[36] A. Francis, O. Kaczmarek, M. Laine, T. Neuhaus and H. Ohno, Critical point and scale setting in SU(3) plasma: an update, Phys. Rev. D 91 (2015) 096002 [arXiv:1503.05652] [INSPIRE].

[37] M. Pospelov and A. Ritz, Astrophysical signatures of secluded dark matter, Phys. Lett. B 671 (2009) 391 [arXiv:0810.1502] [INSPIRE].

[38] M. Ibe, H. Murayama and T.T. Yanagida, Breit-Wigner enhancement of dark matter annihilation, Phys. Rev. D 79 (2009) 095009 [arXiv:0812.0072] [INSPIRE].

[39] J.D. March-Russell and S.M. West, WIMPonium and boost factors for indirect dark matter detection, Phys. Lett. B 676 (2009) 133 [arXiv:0812.0559] [INSPIRE].

[40] W. Shepherd, T.M.P. Tait and G. Zaharijas, Bound states of weakly interacting dark matter, Phys. Rev. D 79 (2009) 055022 [arXiv:0901.2125] [InSPIRE].

[41] T. Hur and P. Ko, Scale invariant extension of the Standard Model with strongly interacting hidden sector, Phys. Rev. Lett. 106 (2011) 141802 [arXiv:1103.2571] [InSPIRE].

[42] J.M. Cline, Z. Liu, G. Moore and W. Xue, Composite strongly interacting dark matter, Phys. Rev. D 90 (2014) 015023 [arXiv:1312.3325] [InSPIRE].

[43] Y. Hochberg, E. Kuflik, T. Volansky and J.G. Wacker, Mechanism for thermal relic dark matter of strongly interacting massive particles, Phys. Rev. Lett. 113 (2014) 171301 [arXiv: 1402.5143] [INSPIRE].

[44] G.D. Kribs and E.T. Neil, Review of strongly-coupled composite dark matter models and lattice simulations, arXiv:1604.04627.

[45] D. Banerjee, S. Datta, R. Gavai and P. Majumdar, Heavy quark momentum diffusion coefficient from lattice QCD, Phys. Rev. D 85 (2012) 014510 [arXiv:1109.5738] [INSPIRE].

[46] A. Francis, O. Kaczmarek, M. Laine, T. Neuhaus and H. Ohno, Nonperturbative estimate of the heavy quark momentum diffusion coefficient, Phys. Rev. D 92 (2015) 116003 [arXiv: 1508.04543] [INSPIRE].

[47] A. Dainese et al., Heavy ions at the future circular collider, arXiv:1605.01389 [INSPIRE].

[48] G.P. Lepage and P.B. Mackenzie, On the viability of lattice perturbation theory, Phys. Rev. D 48 (1993) 2250 [hep-lat/9209022] [INSPIRE]. 